
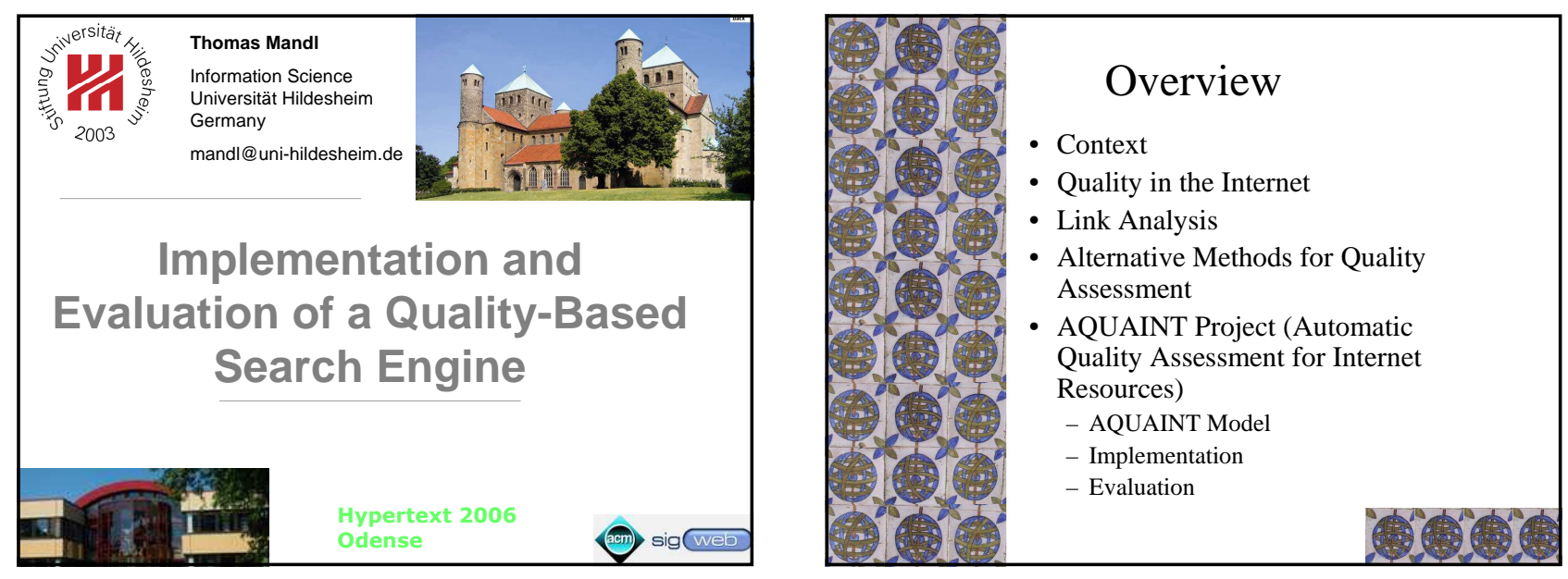

\title{
Lack of Quality on the Internet
}

- "a large fraction of low quality web pages that users are unlikely to read" (Page et al. 1998:2)

- "False infomation abounds, either accidentally or with evil intent" (Weinstein \& Neumann 2000)

- "information quality varies widely on the Internet" (Zhu \& Gauch 2000:288)

\section{Automatic Quality Assessment is Reality}

- Automatic Grading of Essays for College Entry Exams in the USA (Miltsakaki \& Kukich 2004)

- Recommendation Systems: human judgements are aggregated and weighted ba complex algorithms (Avesani et al. 2005)

\section{Framework for Definitions of Quality}

- Transcendent: objective and absolute quality, which is universally valid

- User-oriented: subjektivity, quality depends on context and situation of the user

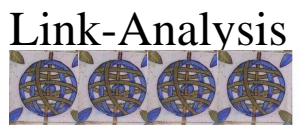




\section{Link-Analysis: Basic Idea}

- Current standard approach to automatic quality assessment

- Basic idea stems from Biblio- or Scientometrics

- Many links to an object support its authority

- Most well known algorithm: PageRank (maybe applied by Google)

\section{Link-Analysis: PageRank}

- The more links pointing to a page, the higher is its authority

- The higher the authority of a page, the more it contributes to the authority of the target page

- Iterative algorithm

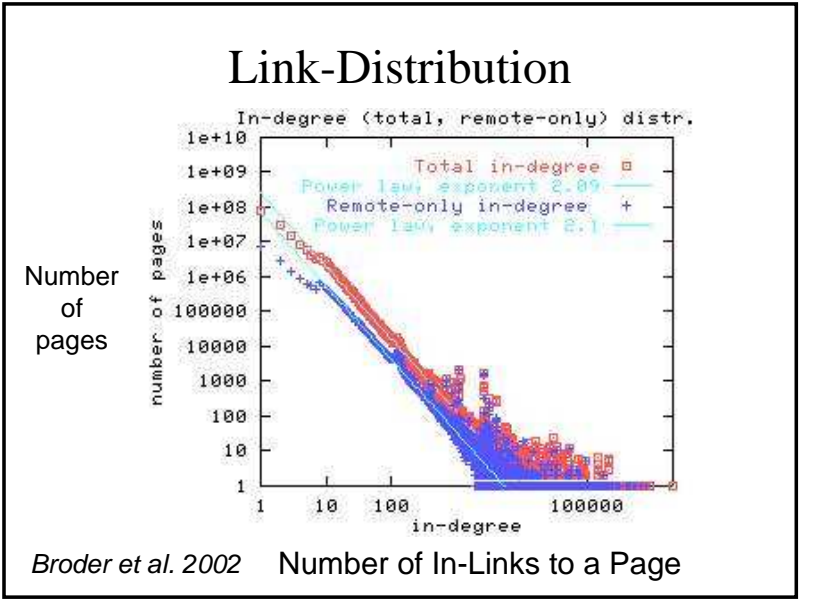

\section{Matthew-Effect}

- Jesus said:

- "For everyone who has will be given more, and he will have an abundance. Whoever does not have, even what he has will be taken from him."

(Matthew 25:29)

\section{Growth Model}

$\Pi(l(i))=\alpha \frac{l c(i)}{L}+(1-\alpha) \frac{1}{U}$

$\Pi(l(i)) \quad$ Pr obability, that new link refers to unit $i$

lc(i) number of in - links of unit i (Link-Count)

$L \quad$ current number of links in the network

$U \quad$ current number of units in the network

$\alpha \quad$ parameter

$a=0.9$ - Effect! (PENNOCK ET AL. 2002:3) 


\section{Web-Track: Results}

- Several groups tested PageRank in the TREC web track

- Improvement could only be noted for the homepage finding task

\section{Link-Analysis}

- Link Analysis is insufficient as the only basis for quality assessment

- experimental systems are searching for alternative approaches

- -> AQUAINT
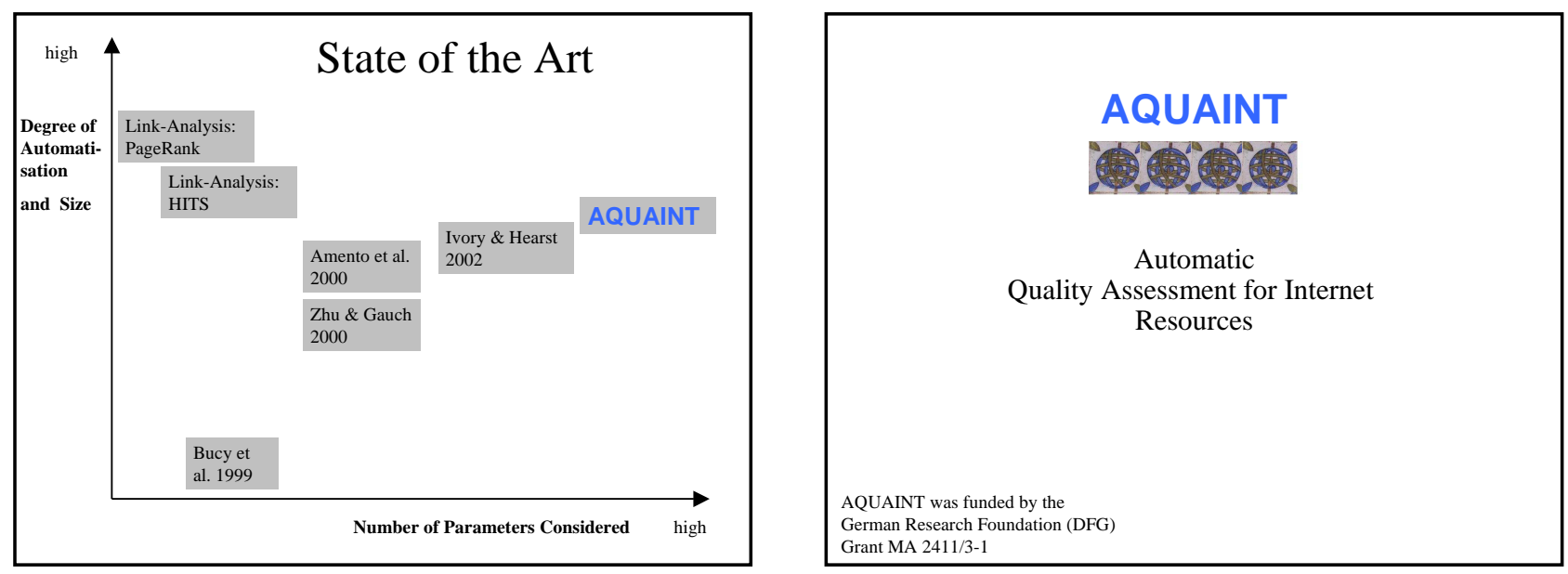

\section{AQUAINT}

- Perspektive: Quality Information Retrieval

- Quality Basis: Decisions made at InternetCatalogues (Yahoo)

- Other web pages as contrastive (negativ) pages

- Different pages are used for model development and for evaluation

- Evaluation considers retrieval effectivity and page quality

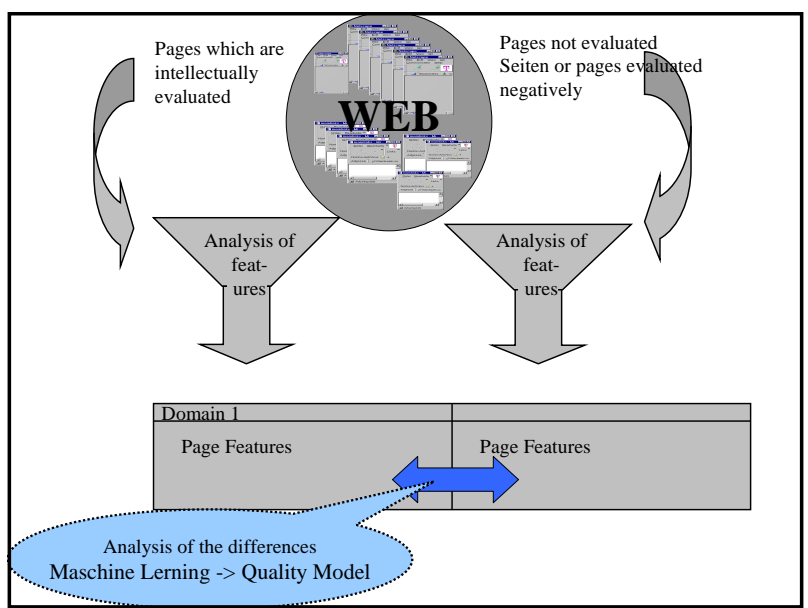




\section{Features}

- Single Features tell us little or are ambivalent

- Example: age of a page

- Conference pages from last year?

- ->Complex Quality Model

- Disadvantage: no transparency

\section{Features: Design}

- Design very important for human quality judgement (Tractinsky 1997, Bouch et al. 2000)

- Eye is primarily directed to graphic elements (Ollermann et al. 2004)

- Strong correlation between design und trust (Fogg et al. 2001)

\section{AQUAINT: Features}

- Features extracted from HTML Code and DOM

- Some 110 features

- Partly from previous research

- Examples for features

- Graphic vs. Text orientation (Colors, Graphics)

- Structure and complexity

- Size of some elements (Tags)

- Text, Links, Hierarchy Level

- Balance (e.g. between Links and Text ...)
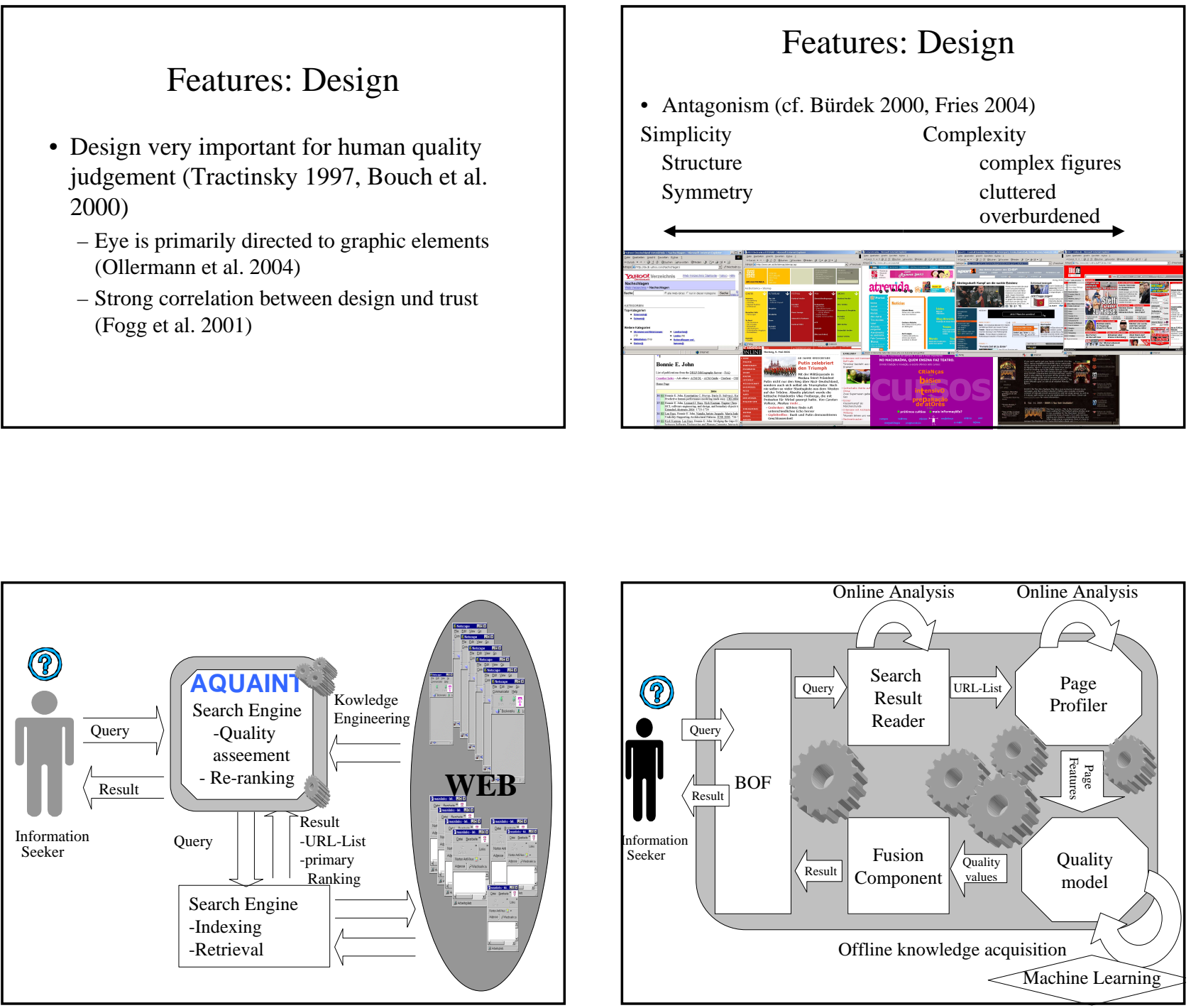


\section{Quality Model}

- Current model

- some 15.000 pages from Yahoo - Health

- some 15.000 pages from Search engines

- some 10.000 intellektually found Spam

(Source: Lycos Europe)

- Linear Regression Model

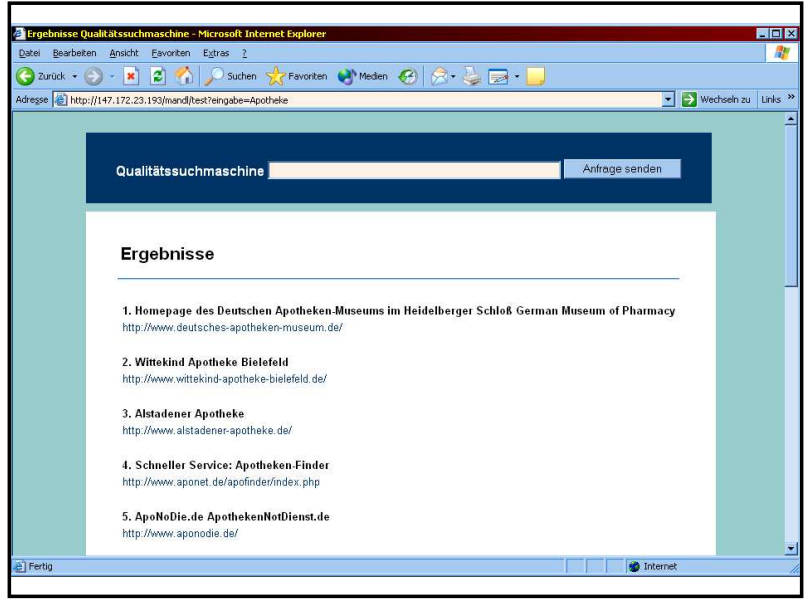

\section{Evaluation}

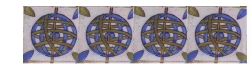

\section{Evaluation}

- Searches in Domain Health

- Grading of results pages by test users

- According to relevance and

- Quality

- 20 test users with 10 queries each

- Log-File

- Notes of test administrators

\section{Evaluation: Subjektivity of Quality Judgements}

- "The quality of a web site inherently is a matter of human judgement"

(Amtento et al. 2000:296)

- "In fact, for a website there can be as many views of its quality as there are usages" (Brajnik 2001:2)

- "Many kinds of human judgement are intrinsically inconsistent" (Mizzaro 1997:814)

\section{Evaluation: Subjectivity of Quality}

\section{-> Break with Cranfield-Paradigm of} Evaluation in Information Retrieval

- No transcendent and absolute relevance

- But individual, subjective quality evaluation in the context

- Different evaluation strategy as in standard information retrieval evaluation (TREC, CLEF, NTCIR, INEX, ...) 


\begin{tabular}{|c|c|c|c|}
\hline \multicolumn{4}{|c|}{$\begin{array}{c}\text { Evaluation Results AQUAINT: } \\
\text { At Ten Documents }\end{array}$} \\
\hline Ranking Method & \begin{tabular}{|l} 
Grade assigned by \\
user
\end{tabular} & Quality Grading & Relevance Grading \\
\hline \multirow{3}{*}{ Original Ranking } & & 29 & 71 \\
\hline & Grade 1 to 2 & 101 & 114 \\
\hline & Grade 1 to 3 & 154 & 143 \\
\hline \multirow{3}{*}{ Quality Ranking } & Grade 1 & 32 & 81 \\
\hline & Grade 1 to 2 & 119 & 129 \\
\hline & Grade 1 to 3 & 185 & 167 \\
\hline \multirow{3}{*}{ Random Ranking } & Grade 1 & 20 & 49 \\
\hline & Grade 1 to 2 & 68 & 81 \\
\hline & Grade 1 to 3 & 114 & 109 \\
\hline
\end{tabular}

\begin{tabular}{|c|} 
Future Work \\
- Future Quality Models? \\
- Probably combinations of link analysis, content \\
analysis as well as presentation analysis \\
- Web-Design Mining as a sub task of Web \\
Mining \\
- e.g. colors (Eibl \& Mandl 2005) or structure \\
(Mandl 2003)
\end{tabular}

Thanks for your
Attention
I am looking forward
to the Discussion

